\title{
Parental awareness of vitamin D for children under five years
}

\author{
C. Robinson, L. K. Pourshahidi, H. McCarthy and M. A. Kerr \\ Northern Ireland Centre for Food and Health, University of Ulster, Coleraine, BT52 1SA
}

The UK Department of Health advises that all children from 6 months to 5 years (y) receive vitamin supplementation, including vitamins $\mathrm{A}, \mathrm{C}$ and $\mathrm{D}$, in the absence of more than $500 \mathrm{ml}$ of infant formula per day ${ }^{(1)}$. Recent data from the National Diet and Nutrition Survey shows that UK children under $5 \mathrm{y}$ are generally meeting vitamin recommendations, with the exception of vitamin $\mathrm{D}^{(2)}$. The UK Healthy Start Scheme (HSS, 2006) includes provision of free vitamin supplements to infants of qualifying families, however uptake of the scheme in Northern Ireland is low ${ }^{(3)}$. The aim of this pilot study was to investigate parental awareness of vitamin D supplementation and the HSS for children under $5 \mathrm{y}$ in Northern Ireland (NI).

An online questionnaire was developed using a web-based survey builder (SurveyMonkey@). Parents/carers $(18 \mathrm{y}+)$, with at least one child under $5 \mathrm{y}$ and living in NI were invited to take part in the study via a local parenting programme, email distribution lists and social networking sites. With respect to their child/children under $5 \mathrm{y}$, parents were asked their perceived importance of vitamin $\mathrm{D}$, the extent of vitamin D advice received from a healthcare professional (HCP), provision of vitamin D containing supplements and awareness of the HSS. General demographic information was also collected.

A total of 47 responses have been collected to-date. The majority of respondents were female (98\%); from an urban area (49\%); married $(72 \%)$; educated to postgraduate level $(38 \%)$ and employed full time $(53 \%)$. A large proportion of respondents regarded vitamin $\mathrm{D}$ as very or extremely important $(67 \%)$, however, only $28 \%$ reported giving their child a vitamin D-containing supplement every day (Figure 1).

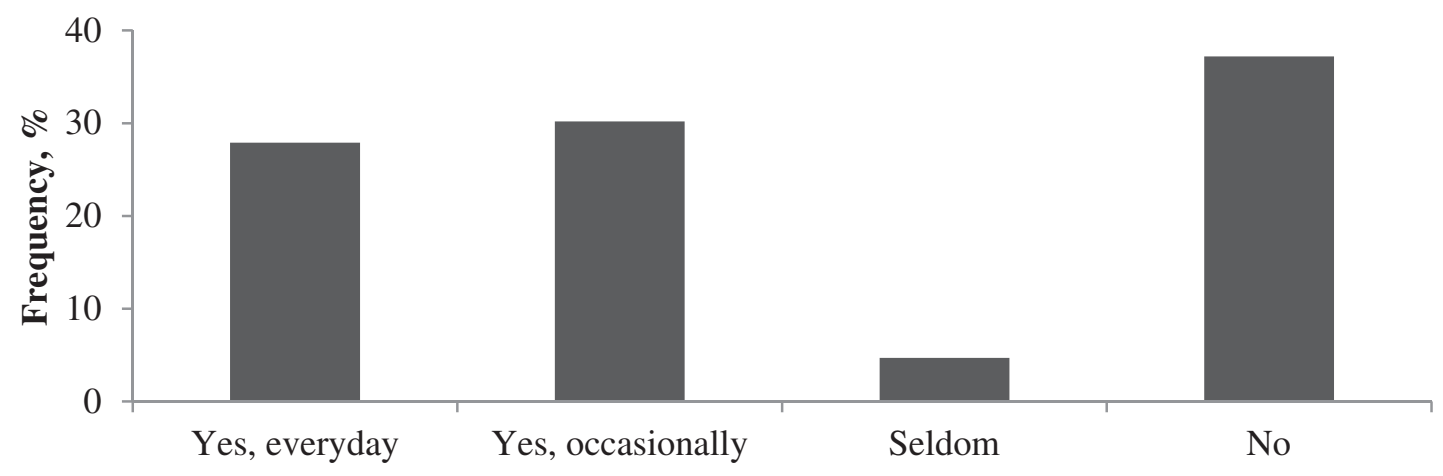

Figure 1. Use of vitamin D supplements in children under 5 years $(n=43)$.

Over three quarters of respondents reported that they had never received advice about vitamin D from a HCP (75\%), and a similar proportion reported that they would like to receive more (72\%). The vast majority of the sample had not heard about the HSS (69\%).

Even in a well-educated sample with a high level of awareness regarding the importance of vitamin D for children under $5 \mathrm{y}$, provision of daily vitamin D supplementation was low. Results from this pilot study suggest inadequate dissemination of vitamin D advice from HCPs, however this must be confirmed in a wider population group. Further research on HCPs knowledge, potential training needs and barriers in relation to dissemination of vitamin $\mathrm{D}$ supplementation in children under $5 \mathrm{y}$ may be warranted.

1. Department of Health. 2012. Vitamin D - advice on supplements for at risk groups. http://www.dh.gov.uk/en/Publicationsandstatistics/Lettersandcirculars/Dearcolleagueletters/DH_132509.

2. Department of Health. 2011. National Diet and Nutrition Survey: Headline results from Years 1 and 2 (combined) of the rolling programme 2008/ 9-2009/10. http://www.dh.gov.uk/en/Publicationsandstatistics/Publications/PublicationsStatistics/DH_128166.

3. Walker A (2007). The Journal of Family Health Care; 17 (2); 53-55. 